\title{
Bacterial Immunotherapy -Antitumoral Potential of the Streptococcal Toxin Streptolysin S-
}

\author{
Claudia Maletzki1 ${ }^{1}$, Bernd Kreikemeyer ${ }^{2}$, Peggy Bodammer ${ }^{3}$, \\ Joerg Emmrich ${ }^{3}$ and Michael Linnebacher ${ }^{1}$ \\ ${ }^{1}$ Department of General, Vascular, Thoracic and Transplantation Surgery, \\ Section of Molecular Oncology and Immunotherapy, \\ 2Institute of Medical Microbiology, Virology and Hygiene, \\ ${ }^{3}$ Division of Gastroenterology Department of Internal \\ Medicine University of Rostock, Rostock,
}

Germany

\section{Introduction}

Chronic infections can lead to cancer. However, acute infection has beneficial effects often contributing to complete eradication of tumors. In the wake of this, bacteria and their related products were applied therapeutically for experimental immunotherapy. They exhibit direct antitumoral potential and are recognized by the host's immune system via Toll-like receptors (TLRs) finally promoting pro-inflammatory, often Th1-directed immune responses.

Recently, we described that local injection of live as well as lysed gram-positive Group A Streptococci (GAS) eradicates established pancreatic tumors in mice (Linnebacher et al., 2008; Maletzki et al., 2008). This antitumoral effect could be attributed to activation of immune response mechanisms including both the innate and even more important, the adaptive arm of the immune system. In the face of the vigorous immune attack induced by S. pyogenes, the identification of factors responsible for tumor disintegration might provide the basis for development of therapeutic approaches. Amongst other virulence factors delivered by S. pyogenes, the cytolysins Streptolysin O (SLO) and S (SLS) represent the most obvious therapeutically active candidates (Fraser \& Proft, 2008; Hobohm et al., 2008; Nizet et al., 2008). SLO is an oxygen-labile, pore-forming toxin mediating cytolysis by disturbing the balance between influxes and effluxes across the cell membrane. While SLS is nonimmunogenic in the natural course of infection and can clinically be identified by betahaemolysis surrounding GAS colonies grown on blood agar. Besides their capacity to lyse erythrocytes, SLS also exerts cytolytic effects towards tumor cells and is by weight one of the most potent cytotoxins known (Ginsberg, 1999; Taketo \& Taketo, 1966).

To address the question of the SLS contribution to the antitumoral effects observed in our previous studies, we performed a series of in vivo experiments in our murine syngeneic 
Panc02 tumor model using different strategies of SLS-inactivation. Endpoints of the study were survival and tumor response. In a first series of experiments, a vital mutant strain, unable to produce SLS $(\Delta$ sagA) was injected into tumor-carrying mice. This $\Delta$ sagA mutant has been described to exhibit reduced cytotoxicity. In vitro and in a nude mouse model in vivo, effects were induced by minimal inflammation and lesser necrotic skin lesions than the isogenic wildtype strain (Datta et al., 2005). However, to circumvent the potential risk of unpleasant side effects of live bacteria such as systemic toxicity, another microbial preparation of SLS-inactivated bacteria (by heating) was employed. Our in vivo data show that local application of SLS-negative agents attenuates the antitumoral effects. Moreover, potent immune responses are only marginally induced, most likely because of reduced tumor cell impairment after infection, finally leading to an inhibition of vigorous antitumoral activity.

In summary, when comparing with our earlier findings on using $S$. pyogenes as an active immunotherapeutic compound, the present data imply SLS as major antitumoral molecule both directly by acting on tumor cells and indirectly by activating the immune system.

\section{Material \& methods}

\subsection{Cell culture \& mice}

All cell lines (Panc02, EL4, CMT-93, and MC3T3-E1) were maintained in DMEM/HamsF12 supplemented with $10 \%$ fetal calf serum (FCS), L-glutamine ( $2 \mathrm{mmol} / \mathrm{l})$ and antibiotics. All media and supplements were from PAA unless stated otherwise (Cölbe, Germany). Female 8-10-week-old C57Bl/6N mice were purchased from Charles River Inc. (Sulzfeld, Germany). Animals were exposed to cycles of $12 \mathrm{~h}$ light/12 h darkness and received standard food and water ad libitum. Upon approval by the local animal welfare committee, experiments were performed in accordance with the German legislation on protection of animals and the "Guide for the Care and Use of Laboratory Animals".

\subsection{Bacteria, toxin and culture conditions}

Bacteria (S. pyogenes serotype M49 strain 591; the $\Delta$ sagA mutant isogenic to strain 591 is a kind gift from Victor Nizet: A detailed description of how this mutant was generated can be found in (Datta et al., 2005)) were cultured in Todd-Hewitt (TH) broth or on TH agar (Oxoid Unipath, Wesel, Germany), both supplemented with $0.5 \%$ yeast extract (THY) overnight to mid-log phase. Concentration was adjusted on the basis of an optical density reading at 600 $\mathrm{nm}$ and on plating analysis. Heat-inactivation of bacteria was obtained by one-hour incubation in a water bath at $75^{\circ} \mathrm{C}$. Inactivation was confirmed by plating samples on sheep blood agar followed by over night incubation at $37^{\circ} \mathrm{C}$ and $5 \% \mathrm{CO}_{2}$. The purified toxin SLS (originally obtained from Sigma Aldrich, Munich, Germany) was dissolved in sterile PBS and supplemented with complete cell culture medium before treatment of cells.

\subsection{Hemolysis assay for SLS activity}

Hemolytic activity of SLS was determined by hemoglobin release from whole blood cells after 4 and $24 \mathrm{~h}$ incubation with bacteria or their related products. Briefly, whole blood of healthy donors was seeded in 96-well plates and supplemented with microbia. Following 
the incubation time, cell-free supernatants were transferred into a new 96-well plate and absorption was measured on a plate reader at $340 \mathrm{~nm}$. Hemolytic activity was quantified by setting values of untreated cells as 1 and all other data were given as $x$-fold increase.

\subsection{Cellular cytotoxicity assays}

Toxicity of bacteria towards tumor cells was quantified using a cytotoxicity detection kit (Roche, Mannheim, Germany) according to the manufacturer's instructions. Quantification was performed by subsequent measurement on a plate reader at $492 \mathrm{~nm}$.

For detection of apoptosis, activity of Caspase 3 was analyzed using the BD ApoAlert Caspase Assay plate system according to the manufacturer's instructions (BD Biosciences, Heidelberg, Germany). Cells were trypsinized and subsequently lysed. Quantification of Caspase 3 activity was performed by fluorometric detection on a Cytofluor 2300 (Millipore, Schwalbach, Germany, ex/em: 380/460 nm).

\subsection{Pancreatic tumor model \& treatment regimen}

Under brief ether anaesthesia $1 * 10^{6}$ Panc02 were injected subcutaneously (s.c.) into the right hind leg. Tumor growth was routinely controlled at least twice a week and tumor volume was estimated according to the formula: $\mathrm{V}=$ width $^{2} *$ length $* 0.52$. After tumor establishment animals were subdivided into experimental groups. All treatments were performed by local, intratumoral application of bacterial preparations each dissolved in $50 \mu \mathrm{l}$ of phosphate-buffered saline (PBS) according to the following treatment regimen: One group was given heat-inactivated S. pyogenes $\left(8 \times 10^{7} \mathrm{cfu}\right.$, four injections once a week, $\left.\mathrm{n}=6\right)$. The second group received a single injection of the SLS-mu $(\Delta$ sagA). For control, mice were administered equivalent volumes of solvent alone (vehicle-treated controls, saline, $\mathrm{n}=6$ ) or left without treatment (control, $n=6$ ). Tumor carrying mice (treatment, control) were sacrificed at day 28 or when they became moribund before the tumor volume reached 2000 $\mathrm{mm}^{3}$. At the end of each experiment, tumors as well as spleens, mesenteric lymph nodes, and blood samples were removed from selected animals for further analysis. For visualization of tumor-infiltrating leukocytes, frozen sections of resected tumor tissues (6 $\mu \mathrm{m})$ were stained by the As-D chloroacetate esterase (As-D) technique. Numbers of leukocytes $/ \mathrm{mm}^{2}$ were determined in blinded counts by positive staining and morphology in 20 consecutive high power fields (HPF).

\subsection{Phenotyping of lymphocyte subpopulations by flow cytometry}

Leukocytes from treated and non-treated animals were labeled using the following FITCconjugated rat anti-mouse monoclonal antibodies (mAbs): CD3, CD19, NK1.1 (1 $\mu \mathrm{g}$, Immunotools, Friesoythe, Germany), and Phycoerythrin (PE)-conjugated rat anti-mouse mAbs: CD4, CD8 (Miltenyi Biotec, Bergisch Gladbach, Germany). Afterwards, erythrocytes were lysed using FACS Lysing Solution (BD Pharmingen, Heidelberg, Germany). Negative controls consisted of blood lymphocytes stained with the appropriate isotypes (BD Pharmingen). Samples were analyzed on a FACSCalibur Cytometer (BD Biosciences). Data analysis was performed using CellQuest software (BD Biosciences) and gating on total leukocytes (Mounting View, BD Biosciences). Relative numbers are given. 


\subsection{ELISPOT assay for Interferon-y-secreting lymphocytes}

IFN- $\gamma$-specific, mAb (Mabtech, Hamburg, Germany) -coated, 96-well microtiter plates were filled with $1 \times 10^{4}$ target cells/well (Panc02, EL4, CMT-93, MC3T3-E1, and peripheral blood mononuclear cells (PBMC)) and incubated for $2 \mathrm{~h}$. Splenocytes $\left(10^{5}\right)$ were given to the targets and co-cultured overnight. Finally, bound antibody was visualized by BCIP/NBT (KPL, Gaithersburg, Maryland, USA), and spots were counted using a dissection microscope (Zeiss, Oberkochen, Germany). Presented are the numbers of IFN- $\gamma-$ secreting cells per $10^{5}$ effector cells corrected for background levels counted in the absence of target cells, which usually was between $10-50$ spots $/ 10^{5}$ cells. Target cells without effector cells showed no background level.

\subsection{LDH cytotoxicity assay}

The colorimetric CytoTox-One Homogeneous Membrane Integrity Assay (Promega, Madison, WI) was used evaluating lactate dehydrogenase (LDH) release from lysed cells. Experiments were performed as described before (Maletzki et al., 2008).

\subsection{Bio-plex protein array system}

A panel of serum cytokines was measured in duplicate using the Bio-Plex Protein Array system (BioRad, Munich, Germany), according to the manufacturer's instructions. With the Bio-Plex cytokine assay kit in combination with the Bio-Plex Manager Software, serum IFN$\gamma$, TNF-a, IL-6, IL-10, GM-CSF (granulocyte macrophage colony stimulating factor), and GCSF (granulocyte-colony stimulating factor) levels were assessed. Values of the respective serum cytokine levels of untreated control mice were set as 1, and all other data were given as $x$-fold increase.

\subsection{Statistical analysis}

All values are expressed as mean \pm SEM. After proving the assumption of normality, differences between saline and treated animals were determined by using the unpaired Student's $t$-test. If normality failed, the nonparametric Mann-Whitney $U$-Test was applied. Similarly, differences between treated and non-treated cell in vitro were calculated by using the nonparametric Mann-Whitney $U$-Test. Data were recruited from experiments which had been done in triplicates and replicated at least three times. The tests were performed by using Sigma-Stat 3.0 (Jandel Corp, San Rafael, CA). The criterion for significance was set to $\mathrm{p}<0.05$.

\section{Results}

\subsection{In vitro analysis}

First, activity of SLS from different bacterial preparations was analyzed in a simple hemoglobin release assay (Figure 1). As expected, the purified toxin mediated substantial lysis of erythrocytes within a few hours. In contrast, lysis was completely absent post infection with the $\Delta \mathrm{sagA}$ strain or the heat-inactivated form of the M49wt, thereby confirming inactivation of the toxin. As a control, vital M49 bacteria and the lysate, used in our previous studies (Maletzki et al., 2008), were employed. Both preparations mediated nearly complete lysis of erythrocytes. 


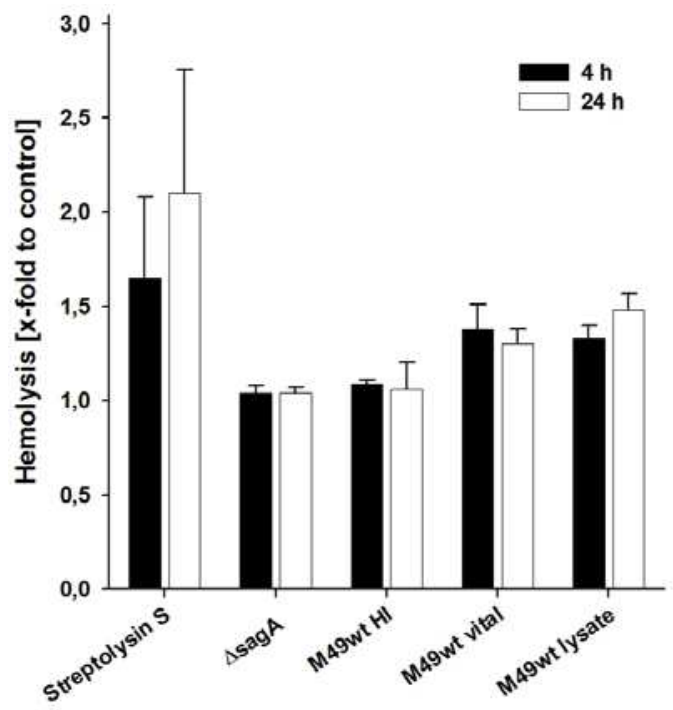

Fig. 1. Lytic activity of different bacterial preparations towards erythrocytes in whole blood samples. Following treatment with increasing concentrations of bacteria, cell-free supernatants were harvested and absorption was measured on a plate reader at $340 \mathrm{~nm}$. Lytic activity was quantified by setting values of untreated cells as 1 , and all other data were given as $x$-fold increase. Results show data from at least three separate experiments each performed with two healthy volunteer's blood samples. Values are given as the mean \pm SEM.

In order to elucidate, if SLS also directly damages tumor cells, we applied the purified toxin in $\mathrm{LDH}$ release experiments. In particular, Panc02 tumor cells were treated with three increasing concentrations $(25,50$, and $100 \mathrm{U} / \mathrm{ml})$ for six hours. These analyses revealed a dose-dependent increase in cell damage with a maximum of up to $65 \%(100 \mathrm{U} / \mathrm{ml})$. To a minor part, these effects could be attributed to the induction of apoptosis as the activity of the effector caspase 3 slightly increased after treatment with the $100 \mathrm{U} / \mathrm{ml}$ doses of SLS (Figure 2).

In line with these findings, a $\Delta$ sagA strain of S. pyogenes mediated no significant growth inhibition or killing activity at a multiplicity of infection of 25 for four and six hours (Figure 2). Also, activity of Caspase 3 was not altered post infection.

\subsection{SLS-deficient preparations of S. pyogenes have minor antitumoral potential}

To further clarify the antitumoral potential of the toxin SLS a series of in vivo experiments using two different preparations of inactivated SLS was performed (Figure 3a). Panc02 tumors established subcutaneously in $\mathrm{C} 57 \mathrm{Bl} / 6$ mice were infected with the $\Delta$ sagA strain $\left(10^{7} \mathrm{cfu}\right)$. The intratumoral administration of $\Delta$ sagA did not affect pancreatic carcinoma growth within the first three weeks. Palpable tumors continued to grow and reached an average size of $723.8 \pm$ $95.8 \mathrm{~mm}^{3}$, which was comparable to tumor sizes of control animals (saline: $841.4 \pm 96.3 \mathrm{~mm}^{3}$ ). Thereafter, tumors in the infection group became frequently ulcerous and necrotized. This finally resulted in about $40 \%$ reduced tumor volumes compared to saline-treated animals (day 28: $689.2 \pm 119.8 \mathrm{~mm}^{3}$ vs. saline: $\left.1228.1 \pm 220.2 \mathrm{~mm}^{3}, \mathrm{p}<0.05\right)$. 

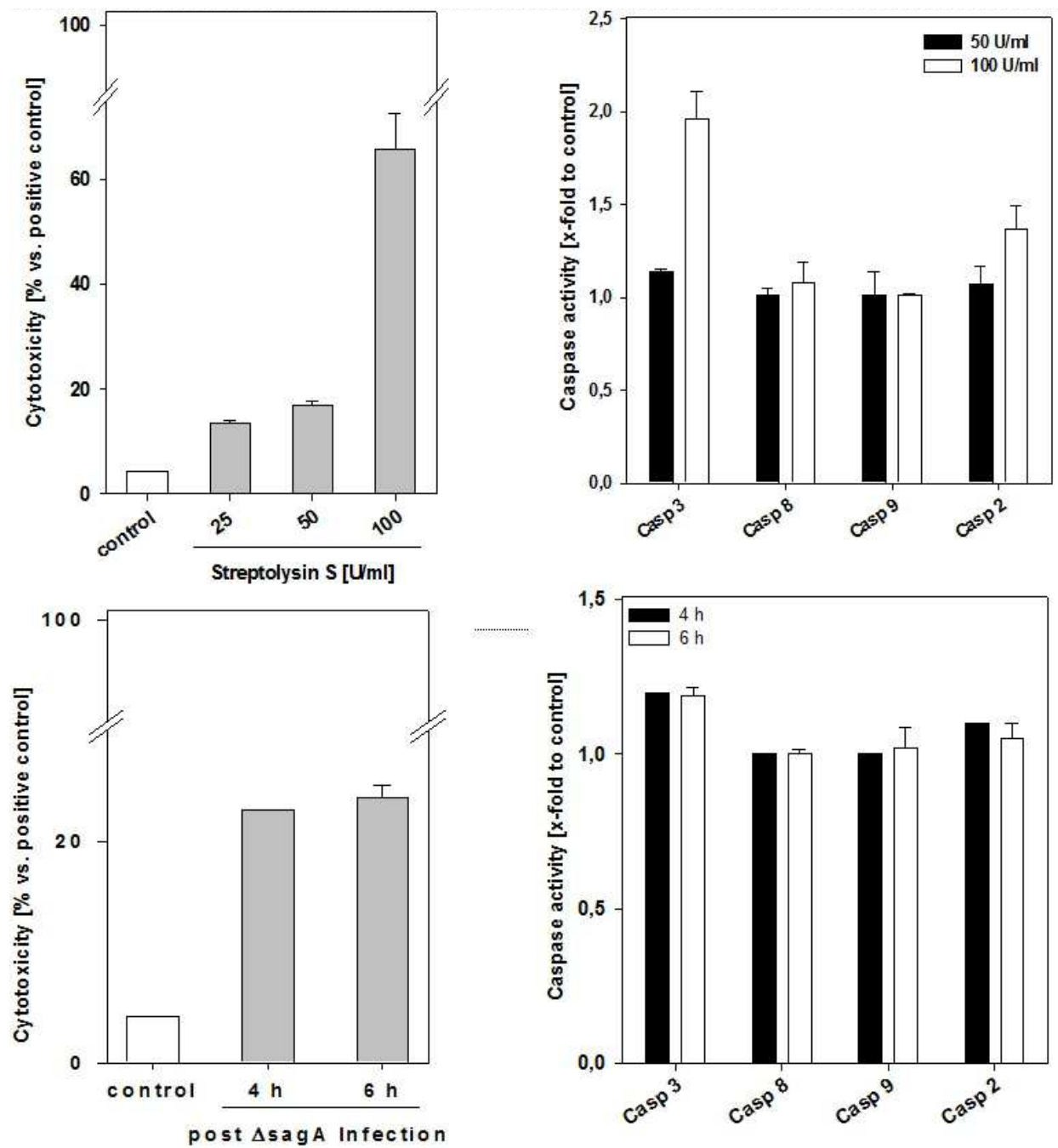

Fig. 2. In vitro analysis on direct effects of SLS on tumor cells. (A) Quantitative analysis of SLS cytotoxicity towards Panc02 cells as assessed by either LDH release or caspase activity following a $6 \mathrm{~h}$ incubation period. These analyses revealed a dose-dependent increase in cell damage. (B) Quantitative analysis of the $\Delta$ sagA effects on Panc02 tumor cells. Infection with the $\Delta$ sagA strain mediated no significant cytotoxicity, detected by LDH release. Results show data from three separate experiments. Values are given as the mean $\pm S E M .{ }^{*} p<0.05$ vs. control, U-Test.

To validate these findings, tumor-carrying animals were repeatedly treated with a bacterial preparation which was preheated to inactivate SLS. Similar to what has been observed after i.t. infection with the $\triangle$ sagA strain, injection of heat-inactivated streptococci mediated no significant alteration of Panc02 tumor growth until day 14 post start of therapy. However, 
when comparing to controls, tumors showed a trend towards growth retardation, suggesting only reduced but not completely abolished antitumoral potential of the heatinactivated bacteria. Hence, the final tumor volume at day 28 was significantly lower than in saline-treated mice $\left(693.2 \pm 63.0 \mathrm{~mm}^{3}\right.$ vs. $\left.1154.5 \pm 277.1 \mathrm{~mm}^{3}, \mathrm{p}<0.05\right)$.

\subsection{Survival}

Animals were sacrificed at day 28 post start of therapy. Infection with the $\Delta$ sagA strain was well tolerated by most animals, with only one animal displaying signs of systemic bacterial infection (i.e. weight loss, ataxia). Because of the severity of infection, this animal was euthanized and related data excluded. As expected, the heat-inactivated bacterial preparation had no negative impact on survival (Figure 3b).
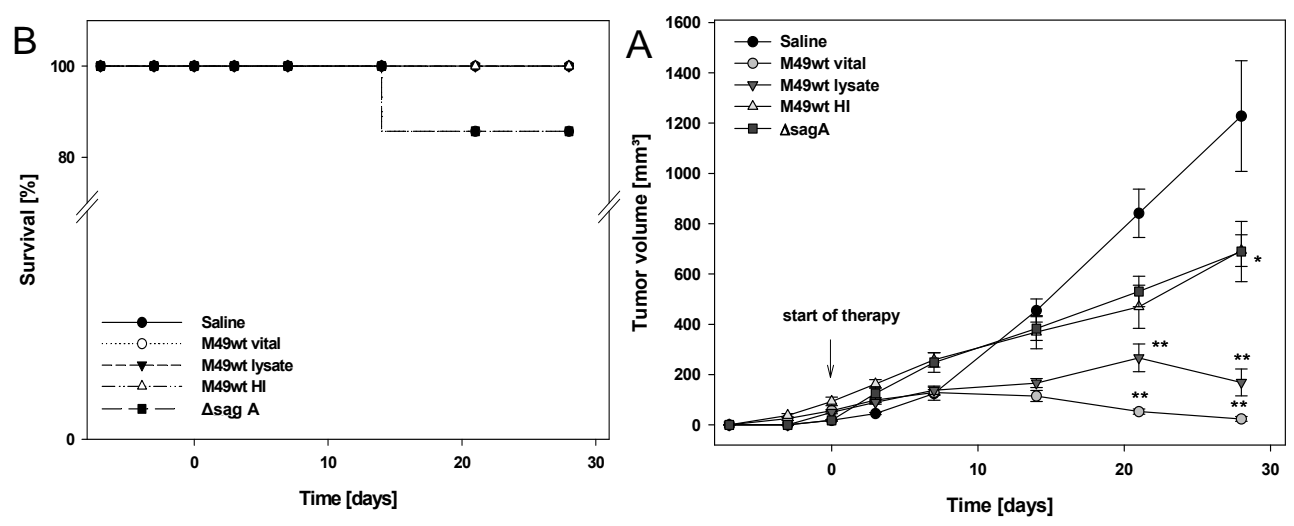

Fig. 3. Data of in vivo analysis of Panc02-tumor carrying C57B1/6N mice. (A) Tumor growth kinetics and (B) survival curve after i.t. application of bacteria. Treatment regimens comprised the vital SLS-deficient $\Delta$ sagA strain $\left(1 \times 10^{7} \mathrm{cfu}\right.$, ), which is isogenic to the M49 wildtype and heat-inactivated preparations of the M49 wildtype $\left(8 \times 10^{7} \mathrm{cfu}\right.$, four injections once a week, $\mathrm{n}=6$ ). Control mice were administered equivalent volumes of solvent alone (saline, $\mathrm{n}=6$ ) or left without treatment (tumor, $n=6$ ). Animals were sacrificed at day 28 or when they became moribund before the tumor volume reached $2000 \mathrm{~mm}^{3}$. Tumor growth was only at later stages affected and when comparing with our previous studies on using vital as well as lysed wild type bacteria, to a lesser extend (graphical presentation adopted from Linnebacher et al., 2008 and Maletzki et al., 2008). ${ }^{*} p<0.05$ vs. saline, ${ }^{* *} p<0.001$ vs. saline $U$-Test.

\subsection{Gross findings \& hematological alterations post $\Delta$ sagA infection}

To further validate the potential of SLS to influence tumor growth, we analyzed systemic parameters of animals treated with $\Delta$ sagA in more detail. Analysis of blood samples from $\Delta$ sagA infected animals revealed no alteration regarding the number of circulating leukocytes. However, thrombocyte as well as hematocrit levels were found to be decreased post infection (Figure 4a). Similarly, activities of the plasma enzymes ASAT and LDH were reduced, while levels of ALAT were not affected by bacteria (Figure $4 \mathrm{~b}$ ). In this case, the infection-mediated decrease in blood LDH activities might correlate with the retardation of tumor growth observed in vivo. 
As these data indicated an inflammatory reaction in the $\Delta$ sagA treated animals, we subsequently determined plasma cytokine levels from infected and non-infected animals (Figure 4c). Analysis of the neutrophile chemotaxis polypeptide G-CSF and the GM-CSF showed slight increases post infection which were, however not statistically significant. Levels of both cytokines displayed 2-fold rises post infection ( $p=0.256$ vs. saline (G-CSF) and $\mathrm{p}=0.007$ vs. saline (GM-CSF), $t$-test). A similar pattern was seen for IL6 ( $p=0.62)$, while the Th1 cytokines TNF- $\alpha$ and IFN- $\gamma$ showed no alterations at all.
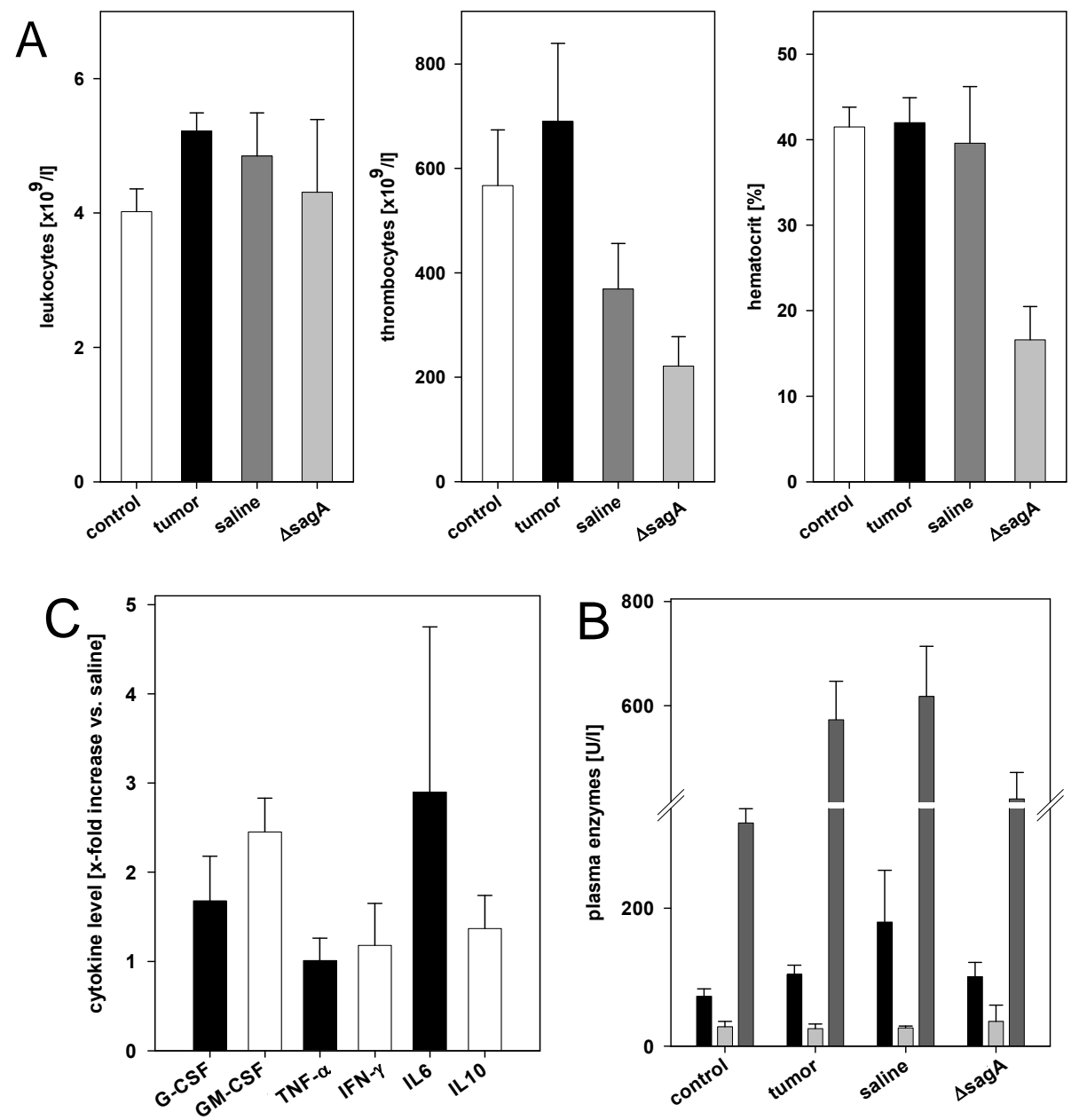

Fig. 4. Assessment of systemic blood parameters. (A) Numbers of leukocytes, thrombocytes, and hematocrit levels as well as (B) plasma enzyme activities of ALAT, ASAT and LDH from $\triangle$ sagA-infected and control animals at day 28. (C) Levels of $T h_{1}$ and $\mathrm{Th}_{2}$ cytokines in serum of treated and untreated mice. Mice received a single i.t. infection of the $\triangle$ sagA strain ( $\left.1 \times 10^{7} \mathrm{cfu}\right)$. Control animals received equivalent volumes of PBS (saline) or no injection (tumor). Values are given as the mean \pm SEM. $U$-Test. 


\subsection{Flow cytometric phenotyping of circulating leukocytes}

Next, flow cytometric phenotyping of circulating leukocytes was performed. As shown in Table 1, we observed higher numbers of circulating NK cells in animals treated with the $\Delta$ sagA strain $(34.6 \pm 7.1 \%$ vs. saline $16.5 \pm 2.7 \%)$. Similarly, levels of Gr1+ granulocytes were also raised $(32.5 \pm 4.6 \%$ vs. saline $25.0 \pm 3.0 \%)$. Regarding the number of $\mathrm{T}$ cells, no significant differences were obtained between the infection and control groups. Likewise, numbers of circulating $\mathrm{CD} 19^{+}$pre $\mathrm{B}$ cells showed no alteration after therapy with values remaining similar to controls. Thus, in our experimental system, microbial therapy with SLS-deficient or heat-inactivated S. pyogenes preparations seemed to affect exclusively the innate arm of the immune system.

\begin{tabular}{lcccc}
\hline & Control & Tumor & Saline & ssagA \\
\hline CD3 $^{+}$CD4 $^{+}$ & $19.5 \pm 1.9$ & $15.7 \pm 1.4$ & $8.6 \pm 1.4$ & $14.1 \pm 0.9$ \\
CD3 $^{+}{ }^{+}$DD $^{+}$ & $10.9 \pm 0.7$ & $9.5 \pm 1.3$ & $6.9 \pm 1.7$ & $10.3 \pm 0.5$ \\
CD19 $^{+}$ & $56.5 \pm 1.9$ & $42.2 \pm 4.1$ & $31.4 \pm 2.5$ & $41.6 \pm 4.2$ \\
NK1.1 $^{+}$ & $14.8 \pm 2.6$ & $15.8 \pm 1.8$ & $16.5 \pm 2.7$ & $34.6 \pm 7.1$ \\
Gr1 $^{+}$ & $9.5 \pm 2.4$ & $19.8 \pm 3.1$ & $25.0 \pm 3.0$ & $32.5 \pm 4.6$ \\
\hline
\end{tabular}

Values are given as mean \pm SEM. $\Delta$ sagA-infected animals $(n=7)$; saline-treated animals $(n=6)$; tumor control animals $(\mathrm{n}=7)$; U-Test.

Table 1. Flow cytometric phenotyping of whole blood in control groups (control, tumor, saline) and post infection with the $\Delta$ sagA strain ( $\%$ positive cells).

\subsection{Analysis of antitumoral immune responses}

Despite raised levels of infiltrating granulocytes, especially in the boundary areas of treated tumors (Figure 5a), we did not observe any significant difference in the number of $\mathrm{CD}^{+}$and CD8+ lymphocytes infiltrating tumors between control and treatment groups (data not shown).

In a first series of experiments, reactivity against syngeneic tumor cell lines was tested in ELISpot assays using lymph node derived lymphocytes as effector cells from control as well as $\Delta$ sagA-treated animals. Surprisingly, recognition of tumor cells was restricted to the syngeneic colorectal carcinoma cell line CMT-93. Other tested cells (i.e. Panc02, EL4) did not induce release of IFN- $\gamma$ from lymphocytes of $\Delta$ sagA treated animals (Figure $5 b$ ).

In a more functional cytotoxicity assay, splenocytes were used as effectors. Again, Panc02 tumor cells were only ineffectively lysed by immune cells from infected mice $(22.1 \pm 2.5 \%$ vs. saline $6.7 \pm 1.7 \%)$. Similar results were obtained with the non-cancerous MC3T3-E1 fibroblasts and with PBMCs (Figure 5c), indicating only little specific killing activity of effector cells. In contrast, we again observed most lytic activity against the syngeneic tumor cell line CMT-93 (35.8 $\pm 12.7 \%$ vs. saline: $22.2 \% \pm 7.5 \%$ ) and additionally against EL4 (38.6 \pm $11.4 \%$ vs. saline: $18.2 \% \pm 3.0 \%)$. 

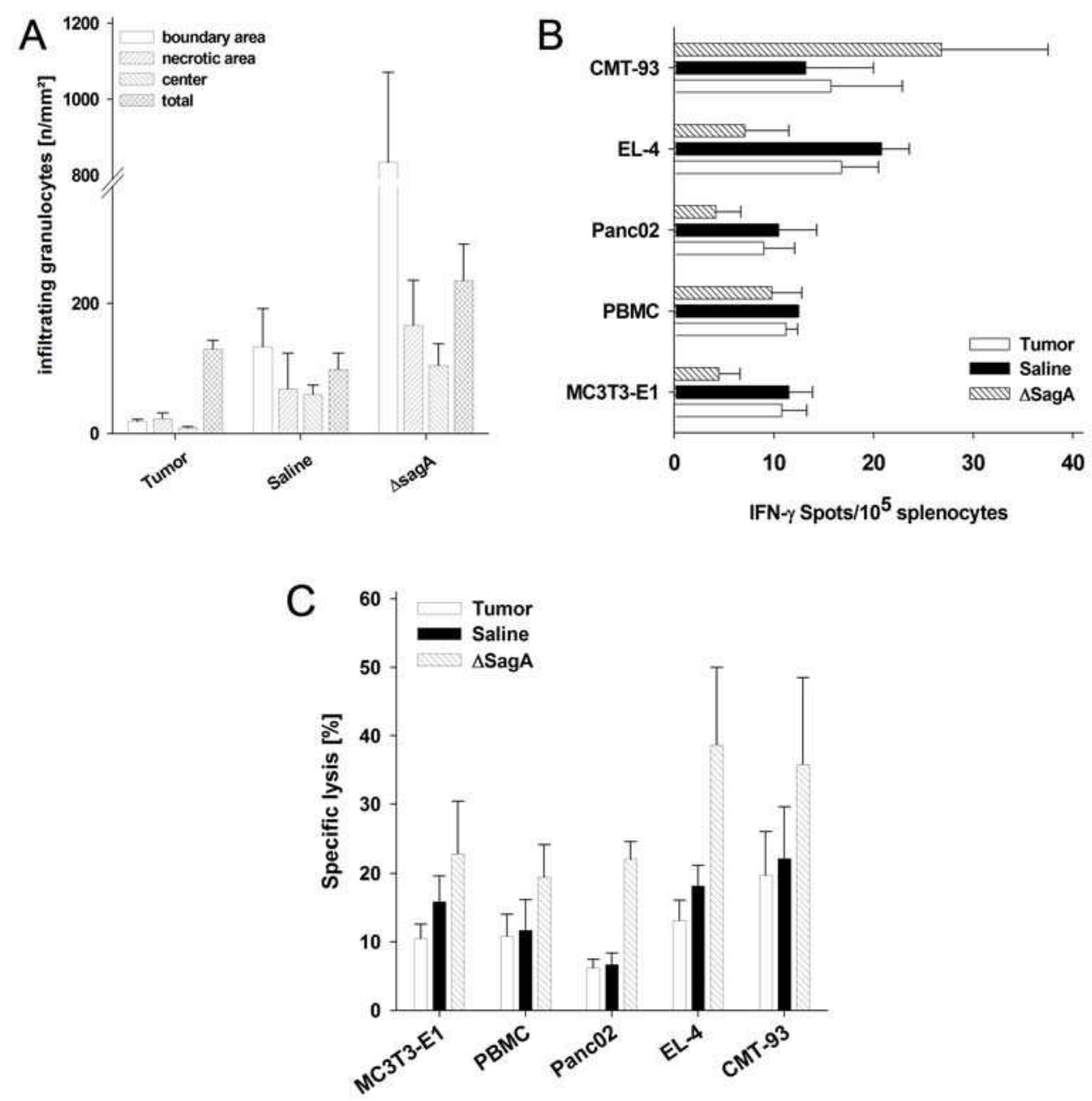

Fig. 5. Data of immune responses from Panc02 tumor-carrying mice. (A) Quantitative analysis of tumor-infiltrating As-D chloroacetate esterase-positive leukocytes shown as number of positive cells per $\mathrm{mm}^{2}$. Leukocytes were found especially in boundary areas, and minor in the center of treated tumors. (B) Quantitative analysis of IFN- $\gamma$ ELISpot assay. Reactivity of splenocytes against tumor targets was tested after co-incubation overnight at an E:T cell ratio of 30:1. Splenocytes from infected animals at 28 days after i.t. bacterial infection showed a marginally higher reactivity against target cells than those from control animals. (C) Quantitative analysis of cytotoxicity using LDH release assay. Lymphocytes were isolated from mesenteric lymph nodes and co-cultured with targets for $24 \mathrm{~h}$ at an E:T ratio of 30:1. Lymphocytes from infected animals at 28 days after i.t. bacterial infection lysed syngeneic tumor cell lines EL4 and CMT-93, but only to a minor extend Panc02 cells. Experiments were performed in triplicates. Values are given as the mean $\pm S E M$. ${ }^{*} p<0.05$ vs. saline, $U$-Test. 


\section{Discussion}

Different observations indicate that exposure by vaccination or infection to pathogenassociated molecular patterns (PAMP) can have beneficial effects on neoplastic diseases (Hobohm et al., 2008). PAMP recognizing TLRs serve as a first line of defense for the immune system, inducing soluble and cellular mediators of innate immunity and initiating key steps of the adaptive immune response. In recent years, manipulating the immune response via TLR stimulation has gained therapeutic and/or prophylactic value for cancer. In particular, administration of the synthetic TLR9 agonist CpG-ODN is being developed for cancer vaccines and cancer therapy, due to its described capacity to stimulate $T h_{1}$-like innate and adaptive anti-tumor responses in numerous preclinical models (Krieg 2007; Jacobs et al., 2010). Another potent inducer of tumor-directed cellular immune responses is the TLR7 agonist imiquimod. In recent years, several studies proved enhancement of antigen-specific $\mathrm{T}$ cell activation followed by tumor eradication (Rechtsteiner et al., 2005; Prins et al., 2006; Xiong \& Ohlfest, 2011). Very recently, we provided evidence for tumor growth control by avitalized grampositive bacteria. In these experiments, therapeutic application of avitalized bacteria effectively delayed tumor growth accompanied by increased numbers of tumor-infiltrating immune cells mainly belonging to the innate arm of the immune system (Klier et al., 2011).

In line with this, we previously observed that treatment with S. pyogenes leads to pancreatic tumor reduction or even cure by the orchestrated induction of innate and subsequent adaptive antitumoral immune responses (Linnebacher et al., 2008; Maletzki et al., 2008). However, little is known about the nature of active components responsible for this success. We hypothesized, that bacterial toxins are the most obvious candidate molecules to explain the antitumoral activity of S. pyogenes. Here, we explored the potential of the streptococcalelaborated oxygen-stable cytolysin Streptolysin S. In agreement with previous data referring to the broad cytolytic spectrum of SLS, we were able to confirm that it also efficiently kills tumor cells and in particular Panc02 mouse pancreatic carcinoma cells. To a minor part, these effects could be attributed to the induction of caspase-dependent apoptosis. In a first series of in vivo experiments, the SLS-deficient mutant S. pyogenes strain $\Delta$ sagA showed only impaired antitumoral activity. This strain has been described to exhibit strongly reduced epithelial cell killing compared with SLS-producing wildtype strains (Datta et al., 2005; Lin et al. 2009). Moreover, minimal evidence of necrosis and tissue injury is seen post infection with SLS-negative bacteria in murine models of skin lesions (Datta et al., 2005). Similarly, local infection with the $\Delta$ sagA strain exhibited antitumoral potential only at later timepoints post infection in our syngeneic murine Panc02 tumor model. This therapeutic success was significantly weaker than what we observed in our earlier studies using vital as well as lysed preparations of S. pyogenes. The hypothesis that SLS is a major antitumoral-acting molecule could be further substantiated in a second series of in vivo experiments. The application of streptococci depleted of active SLS by heating also resulted only in reduced antitumoral activity even after repetitive local treatments.

However, despite incomplete eradication of tumors, we want to strengthen the fact, that both preparations led to therapeutic responses as detected by significant reduction in tumor volumes. Thus, other factors are likely to contribute to the antitumoral effects of living and lysed S. pyogenes (e.g. M-Protein, superantigens, lipoteichoic acid) (Chau et al., 2009). Moreover, these findings hint towards a significant contribution of the immune system in partial control of tumor growth. 
To understand the underlying immunological effects evoked by an inflammatory reaction in the $\Delta$ sagA treated animals, leading to reduced but not completely abolished antitumoral potential, ex vivo analyses were performed. Our observations imply an ongoing inflammatory response including systemic production of Th1 cytokines such as G-CSF and GM-CSF as well as raised levels of circulating NK cells and granulocytes. Functional analyses revealed recognition of syngeneic tumor cells as detected by IFN- $\gamma$ release from lymphocytes obtained from infected animals. However, these effects were rather supposed to be mediated by activated NK cells than by tumor antigen specific T cells. This finding is additionally supported by the lymphocytes' killing activity especially towards the syngeneic tumor cell lines EL4 and CMT-93, but only marginally towards Panc02 cells.

To explain our findings, we propose that intratumoral injection of SLS-deficient streptococcal preparations leads to minor tumor cell damage driven by cytotoxic activity of other bacterial components. This triggers a comparably weak local inflammatory reaction followed by negligible systemic activation of the immune system. Thus, few specific antitumoral effector cells will be activated which can not totally control tumor growth whereas NK cells are the main effector type population mediating some degree of tumor growth control. The question, whether SLS may be an interesting molecule for tumor therapy will be addressed in ongoing trials.

\section{Conclusion}

Based on our previous findings on Streptococcus pyogenes-mediated eradication of established pancreatic murine tumors, we here elucidated the impact of the cytolytic toxin Streptolysin S on tumors in vitro and in vivo. We were able to show that direct exposure of the toxin to tumor cells results in a dose-dependent increase in cell damage. Contrary, the SLS-deficient $\Delta$ sagA strain showed only minor cytolytic potential. In vivo, a single i.t. injection of the $\Delta$ sagA strain affected pancreatic carcinomas only at later time points. This hints towards -at least partial- growth control of tumors by SLS, since comparable effects were observed following repetitive local applications of SLS-inactivated (by heating) bacteria. This finding is further supported by the histologic observation of $\Delta$ sagA-infected tumors. Those tumors showed slight increases in infiltrating granulocytes. Moreover, we found that recognition and killing of tumor cells was not restricted to Panc02 cells, but also detectable towards other syngeneic tumor entities. Taken together, we here provide clear evidence of strong antitumoral effects of SLS. However, in terms of the delayed, but significant impact on tumor growth in vivo, other factors are likely to contribute to the strong antitumoral effects of wildtype S. pyogenes.

\section{Acknowledgment}

We kindly thank Prof. Victor Nizet for providing the S. pyogenes strain $\Delta$ sagA.

\section{References}

Chau TA, McCully ML, Brintnell W, An G, Kasper KJ, Vinés ED, Kubes P, Haeryfar SM, McCormick JK, Cairns E, Heinrichs DE, Madrenas J. (2009). Toll-like receptor 2 
ligands on the staphylococcal cell wall downregulate superantigen-induced $\mathrm{T}$ cell activation and prevent toxic shock syndrome. Nat Med. 15: 641-8.

Craft N, Bruhn KW, Nguyen BD, Prins R, Lin JW, Liau LM, Miller JF. 2005. The TLR7 agonist imiquimod enhances the antimelanoma effects of a recombinant Listeria monocytogenes vaccine. J Immunol. 175: 1983-1990.

Datta V, Myskowski SM, Kwinn LA, Chiem DN, Varki N, Kansal RG, Kotb M, Nizet V. 2005. Mutational analysis of the group A streptococcal operon encoding streptolysin S and its virulence role in invasive infection. Mol Microbiol. 56: 68195.

Fraser JD, Proft T. The bacterial superantigen and superantigen-like proteins. Immunol Rev. 2008 Oct; 225: 226-43. Review.

Ginsburg I. 1999. Is streptolysin S of group A streptococci a virulence factor? APMIS. 107: 1051-9.

Hobohm U, Stanford JL, Grange JM. 2008.Pathogen-associated molecular pattern in cancer immunotherapy. Crit Rev Immunol. 28: 95-107.

Jacobs C, Duewell P, Heckelsmiller K, Wei J, Bauernfeind F, Ellermeier J, Kisser U, Bauer CA, Dauer M, Eigler A, Maraskovsky E, Endres S, Schnurr M. 2010. An ISCOM vaccine combined with a TLR9 agonist breaks immune evasion mediated by regulatory $\mathrm{T}$ cells in an orthotopic model of pancreatic carcinoma. Int J Cancer. 19. [Epub ahead of print]

Krieg AM. 2007.Development of TLR9 agonists for cancer therapy. J Clin Invest. 117: 1184-94. Review.

Lin A, Loughman JA, Zinselmeyer BH, Miller MJ, Caparon MG. 2009.Streptolysin S inhibits neutrophil recruitment during the early stages of Streptococcus pyogenes infection. Infect Immun. 77: 5190-201.

Linnebacher M, Maletzki C, Emmrich J, Kreikemeyer B. 2008.Lysates of S. pyogenes serotype M49 induce pancreatic tumor growth delay by specific and unspecific antitumor immune responses. J Immunother. 31: 704-13.

Maletzki C, Linnebacher M, Kreikemeyer B, Emmrich J. 2008. Pancreatic cancer regression by intratumoural injection of live Streptococcus pyogenes in a syngeneic mouse model. Gut. 57: 483-91.

Nizet V, Beall B, Bast DJ, Datta V, Kilburn L, Low DE, De Azavedo JC. 2008. Genetic locus for streptolysin $S$ production by group A streptococcus. Infect Immun. 68: 4245-54.

Prins RM, Craft N, Bruhn KW, Khan-Farooqi H, Koya RC, Stripecke R Miller JF, Liau LM. 2006. TheTLR-7 agonist, imiquimod, enhances dendritic cell survival and promotes tumor antigen-specific $\mathrm{T}$ cell priming: relation to central nervous system antitumor immunity. J Immunol. 176: 157-164.

Rechtsteiner G, Warger T, Osterloh P, Schild H, Radsak MP. 2005. Cutting edge: priming of CTL by transcutaneous peptide immunization with imiquimod. J Immunol. 174: 2476-2480.

Taketo Y, Taketo A. 1966.Cytolytic effect of streptolysin S complex on Ehrlich ascites tumor cells. J Biochem. 60: 357-362. 
Xiong Z, Ohlfest JR. (2011) Topical imiquimod has therapeutic and immunomodulatory effects against intracranial tumors. J Immunother. 34: 264-269.

Klier U, Maletzki C, Göttmann N, Kreikemeyer B, Linnebacher M. (2011) Avitalized bacteria mediate tumor growth control via activation of innate immunity. Cell Immunol. 269: 120-127. 


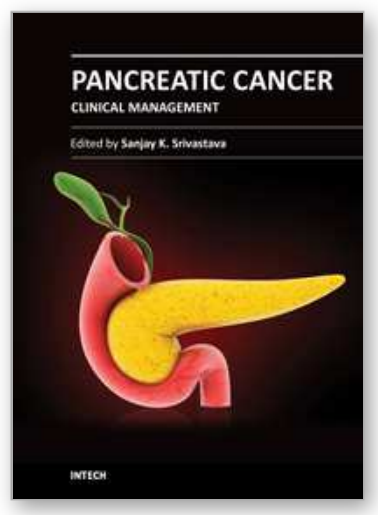

\author{
Pancreatic Cancer - Clinical Management \\ Edited by Prof. Sanjay Srivastava
}

ISBN 978-953-51-0394-3

Hard cover, 312 pages

Publisher InTech

Published online 28, March, 2012

Published in print edition March, 2012

This book covers pancreatic cancer risk factors, treatment and clinical procedures. It provides an outline of pancreatic cancer genetic risk factors, biomarkers and systems biology for the better understanding of disease. As pancreatic cancer suffers from lack of early diagnosis or prognosis markers, this book encompasses stem cell and genetic makers to identify the disease in early stages. The book uncovers the rationale and effectiveness of monotherapy and combination therapy in combating the devastating disease. As immunotherapy is emerging as an attractive approach to cease pancreatic cancer progression, the present book covers various aspects of immunotherapy including innate, adaptive, active, passive and bacterial approaches. Management of anesthesia during surgery and pain after surgery has been discussed. Book also takes the reader through the role of endoscopy and fine needle guided biopsies in diagnosing and observing the disease progression.

\title{
How to reference
}

In order to correctly reference this scholarly work, feel free to copy and paste the following:

Claudia Maletzki, Bernd Kreikemeyer, Peggy Bodammer, Joerg Emmrich and Michael Linnebacher (2012). Bacterial Immunotherapy-Antitumoral Potential of the Streptococcal Toxin Streptolysin S-, Pancreatic Cancer Clinical Management, Prof. Sanjay Srivastava (Ed.), ISBN: 978-953-51-0394-3, InTech, Available from: http://www.intechopen.com/books/pancreatic-cancer-clinical-management/bacterial-immunotherapy

\section{INTECH}

open science | open minds

\section{InTech Europe}

University Campus STeP Ri

Slavka Krautzeka 83/A

51000 Rijeka, Croatia

Phone: +385 (51) 770447

Fax: +385 (51) 686166

www.intechopen.com

\section{InTech China}

Unit 405, Office Block, Hotel Equatorial Shanghai

No.65, Yan An Road (West), Shanghai, 200040, China

中国上海市延安西路65号上海国际贵都大饭店办公楼 405 单元

Phone: +86-21-62489820

Fax: $+86-21-62489821$ 
(C) 2012 The Author(s). Licensee IntechOpen. This is an open access article distributed under the terms of the Creative Commons Attribution 3.0 License, which permits unrestricted use, distribution, and reproduction in any medium, provided the original work is properly cited. 